\title{
PREFERÊNCIA ALIMENTAR DO Tribolium castaneum (COLEOPTERA: TENEBRIONIDAE) EM FARINHA DE SOJA INTEGRAL
}

\author{
Feed preference of Tribolium castaneum (Coleoptera: \\ Tenebrionidae) in Integral Soybean Flour
}

\footnotetext{
Fabiola da Silva Belchol

Especialista em Ciências Ambientais com Ênfase em Gestão Ambiental, Controle de Qualidade - Nutrimental S. A. Ind. e Com. de Alimentos, São José dos Pinhais - PR, E-mail: fbelchol@nutrimental.com.br.

Isabel Ribeiro do Valle Teixeira

Professora do Curso de Pós-Graduação em Gestão Ambiental do Centro Universitário da Fundação Educacional de Guaxupé, MG, Centro Universitário de Guaxupé - UNIFEG, Gaxupé - MG. E-mail: ribeirodovalleteixira@hotmail.com.

Fernanda Freire de Bastos

Eng. Agrônoma, São José dos Pinhais - PR. E-mail: fernandafreirebastos@yahoo.com.br.
}

\begin{abstract}
Resumo
Avaliou-se a preferência alimentar do besouro Tribolium castaneum na farinha de soja integral, sendo que o seu substrato usual é a farinha de milho. Foram utilizados 3 tipos de substratos: farinha de milho, farinha de trigo e farinha de soja integral, placas de Petri com divisória tripla e sem divisórias, estufa entomológica equipada a $32^{\circ} \mathrm{C}$ de temperatura e $70 \%$ de umidade relativa do ar. Foram colocados na placa de Petri com divisória $2 \mathrm{~g}$ de cada substrato e 6 besouros num total de 18 besouros na estufa entomológica, mantidos neste local por 5 dias. Posteriormente, os insetos foram retirados e foi mantida a placa de Petri na estufa entomológica por mais 15 dias para serem contadas as larvas e, entre 21-28 dias, foram observados os adultos emergidos, completando, assim, o ciclo. O mesmo procedimento foi efetuado para a placa de Petri sem divisória, utilizando somente um tipo de substrato. Pode-se observar no experimento que enquanto na farinha de milho e farinha de trigo havia presença de besouros, na farinha de soja havia presença de larvas. As fêmeas foram perfeitamente capazes de ovipositar em outro hospedeiro, desde que esse contivesse os componentes nutricionais necessários para o desenvolvimento, o que permitiu que o inseto se adaptasse a diferentes situações de disponibilidade de alimento.
\end{abstract}

Palavras-chave: Alimentação; Insetos de produtos armazenados; Farinhas. 


\begin{abstract}
It was evaluated the $\square$ feed preference of the beetle ribolium castaneum in the integral soybean flour, despite the usual substrate is the maize flour. Three types of substrates had been used: flour of maize, flour of wheat and flour of integral soybean, Petri dishes with triple partition wall and without partition wall, entomologic greenhouse with temperature of $32^{\circ} \mathrm{C}$ and relative air humidity of $70 \%$. It had been placed in the Petri dishes with partition wall $2 g$ of each substrate and 6 beetles in a total of 18 beetles in the entomologic greenhouse, kept in this place for 5 days. After, the insects had been removed and the Petri dishes kept in the entomologic greenhouse for more 15 days to be counted the larvae and between 21-28 days had been observed the emerged adults thus completing the cycle. The same procedure was affected for the Petri dishes without partition wall only using a type of substrate. It could be observed in the experiment that while in the flour of maize and flour of wheat had presence of beetles, in the soybean flour had presence of larvae. The females were perfectly capable to oviposit in another host, since this contained the necessary nutritional $\square$ components for the development, what it allowed them to adapt to the different situations of food availability.
\end{abstract}

Keywords: Feeding; Stored product insects; Flour.

\title{
INTRODUÇÃO
}

O crescimento populacional de insetos que causam prejuízos aos produtos alimentícios é geralmente mais acelerado que a sua disponibilidade. As perdas quantitativas médias brasileiras, estimadas pela FAO (Food and Drug Administration) e pelo MAPA (Ministério da Agricultura Pecuária e Abastecimento), podem atingir a ordem de 10\% do total produzido a cada ano. Esse quantitativo poderia suprir a carência alimentar anual de 24 milhões de pessoas adultas.

A soja (Glycine Max (L)Merril) é uma leguminosa herbácea anual, possui alto valor protéico em seus grãos (38\%) e fácil adaptação a diversos tipos de clima; é um grão muito versátil, que dá origem a produtos e subprodutos muito usados pelas agroindústrias, indústrias químicas e alimentícias, como: farinhas, lecitina de soja, leite de soja, molhos de soja, gorduras, óleos alimentícios, entre outros produtos (EMBRAPA SOJA, 2003).

O besouro Tribolium castaneum ataca principalmente o milho, porém também ocorrer em outros substratos, como arroz, trigo e o sorgo. Na farinha de soja é comum encontrar a traça Ephestia cautella, vulgarmente conhecida como "traça-do-cacau", que infesta grãos de cereais e seus subprodutos, prejudicando a sua qualidade (LORINI, 2002).

A legislação brasileira define como "farinha" o produto obtido pela moagem da parte comestível de vegetais, podendo sofrer previamente processos tecnológicos adequados. O produto é designado "farinha", seguido do nome do vegetal de origem, como, por exemplo, farinha de trigo, farinha de milho, farinha de soja, farinha de mandioca e entre outros, podendo ser classificada de acordo com as suas características em: a) Farinha Simples: produto obtido da moagem ou raladura dos grãos, rizomas, frutos ou tubérculos de uma só espécie vegetal; b) Farinha Mista: produto obtido pela mistura de farinhas de diferentes espécies vegetais. As farinhas devem ser fabricadas a partir de matérias-primas limpas, isentas de matérias terrosas e parasitos. Não podem estar úmidas, fermentadas ou rançosas (ANVISA, 2001).

A farinha de soja integral é obtida a partir da seleção do grão de soja, conforme Figura 2 e 3 , sendo posteriormente moída e tostada; possui proteínas de alto valor biológico, pois dentre os 20 aminoácidos que o homem necessita, 11 o próprio organismo produz e os outros 9 a soja é capaz de fornecer (ANVISA, 2001).

A farinha de milho foi utilizada no experimento por ser o substrato onde o besouro Tribolium castaneum é mais encontrado.

O objetivo deste trabalho foi observar a preferência alimentar, o desempenho e os aspectos biológicos do besouro Tribolium castaneum em relação aos substratos de farinha de trigo e farinha de soja integral, comparando com o substrato usual farinha de milho. Este trabalho proporciona o conhecimento sobre a interação inseto e a conquista de novos nichos. 


\section{MATERIAL E MÉTODOS}

O experimento foi realizado no laboratório de Biologia do Centro Universitário Educacional de Guaxupé-MG, equipado com uma estufa entomológica de madeira com as medidas 60 x 40 x $40 \mathrm{~cm}$, contendo termostato e resistência elétrica. A metodologia empregada foi desenvolvida por Teixeira e Zucoloto (2003).

Os besouros da espécie Tribolium castaneum foram coletados nos resíduos de farinhas em armazém no município de Arceburgo-MG em fevereiro de 2003.

As amostras de farinha de trigo e farinha de milho foram obtidas de empresas do ramo e a farinha de trigo integral, a farinha de soja integral e a levedura de cerveja foram obtidas no Mercado Municipal de Mococa-SP. As farinhas foram mantidas por 76 horas a $-15^{\circ} \mathrm{C}$ e 6 horas em temperatura ambiente, para eliminar infestações de insetos em todos os seus estágios.

Para acelerar o processo reprodutivo da população de Tribolium castaneum foram utilizados vidros transparentes cobertos com tela tuli e adicionados $10 \mathrm{~g}$ de levedura de cerveja e $40 \mathrm{~g}$ de farinha de trigo integral, sendo denominada como mistura nutritiva.

Foram realizadas quatro repetições de experimentos nos diferentes substratos. Observou-se a preferência alimentar na farinha de soja integral, farinha de milho e farinha de trigo. Realizou-se o método na placa de Petri com divisória tripla e sem divisória contendo o número de 6 carunchos para cada $2 \mathrm{~g}$ de substrato e levados à estufa entomológica a $32^{\circ} \mathrm{C}$ de temperatura e $70 \%$ de umidade relativa do ar.

O desempenho do Tribolium castaneum foi medido usando os seguintes parâmetros: a) Emergência de larvas; b) Preferência alimentar das larvas; c) Preferência alimentar dos adultos. A preferência e aceitabilidade foram medidas por porcentagem de indivíduos em cada substrato.

Foram realizadas 4 repetições de experimentos em placas de Petri com tripla divisória

TABELA 1 - Informação nutricional da farinha de trigo integral e levedura de cerveja denominada como mistura nutritiva

Table 1 - Nutricional information of the integral wheat flour and beer leavening called as Nutritional Mixture

\begin{tabular}{lcc}
\hline Nutrientes & Farinha de Trigo Integral $\mathbf{5 0} \mathbf{g})$ & Levedura de Cerveja (15 g) \\
\hline Valor Calórico & $170,0 \mathrm{kcal}$ & $45,0 \mathrm{kcal}$ \\
Carboidratos & $36,0 \mathrm{~g}$ & $5,0 \mathrm{~g}$ \\
Proteínas & $7,0 \mathrm{~g}$ & $6,0 \mathrm{~g}$ \\
Gorduras Totais & $1,0 \mathrm{~g}$ & $0,0 \mathrm{~g}$ \\
Gorduras Saturadas & $0,0 \mathrm{~g}$ & $0,0 \mathrm{~g}$ \\
Colesterol & $0,0 \mathrm{mg}$ & $0,0 \mathrm{mg}$ \\
Fibra Alimentar & $6,0 \mathrm{~g}$ & $3,0 \mathrm{~g}$ \\
Cálcio & $17,0 \mathrm{mg}$ & $12,0 \mathrm{mg}$ \\
Ferro & $2,0 \mathrm{mg}$ & $3,0 \mathrm{mg}$ \\
Sódio & $0,0 \mathrm{mg}$ & $0,0 \mathrm{mg}$ \\
\hline
\end{tabular}

(oferecendo três tipos de substratos) e sem divisórias (oferecido um tipo de substrato). Foram colocados na placa de Petri com divisória $2 \mathrm{~g}$ dos substratos codificados em A, B e C, sendo A = farinha de milho, B = farinha de trigo e $\mathrm{C}=$ farinha de soja integral, cada um com 6 besouros recém-emergidos da mistura nutritiva, totalizando em cada placa 18 besouros, mantidos na estufa entomológica $(60 \times 40 \times 40 \mathrm{~cm})$ a $32^{\circ} \mathrm{C}$ de temperatura e $70 \%$ de umidade relativa do ar e mantidos neste local por 5 dias. Posteriormente foram retirados os besouros e mantidas as placas de Petri na estufa entomológica por mais 15 dias para serem contadas as larvas e entre 21-28 dias foram observados os adultos emergidos para completar o ciclo de vida. O mesmo procedimento foi efetuado para a placa de Petri sem divisória, utilizando somente um tipo 
de substrato, a fim de observar a aceitabilidade.

A média de larvas que emergiram foi avaliada por meio do número de adultos colocados em cada placa e o número de descendentes, neste caso, larvas originadas em cada substrato.

Para testar a preferência alimentar de larvas de T. castaneum foram realizadas quatro repetições de experimentos com seis besouros na placa de Petri com tripla divisória, com três substratos diferentes: farinha de milho, farinha de trigo e farinha de soja.

TABELA 2 - Emergência de larvas nos substratos com $2 \mathrm{~g}$ de farinha de milho, trigo e soja integral na placa de Petri sem divisória, emergidos com 15 dias

Table 2 - Emergency of larvae in substrata with $2 g$ of maize flour, wheat and integral soybean in the Petri plate without thick partition wall emerged with 15 days

\begin{tabular}{ll}
\hline Tipos de Substratos & o médio de descendentes por indivíduos \\
\hline Milho & $5,96 \pm 0,73$ (a) \\
Trigo & $3,42 \pm 0,22(\mathrm{~b})$ \\
Soja & $1,49 \pm 0,23$ (c) \\
\hline
\end{tabular}

* Letras diferentes indicam diferenças estatísticas (Tukey $P \geq 0,05$ ).

\section{RESULTADOS E DISCUSSÃO}

Percebeu-se uma variação muito grande, quando analisados os diferentes substratos e os tipos de experimentos. Houve menor aceitabilidade na farinha de soja integral e uma correlação positiva

TABELA 3 - Preferência Alimentar de larvas nos substratos com $2 \mathrm{~g}$ de farinha de milho, trigo e soja integral na placa de Petri com divisória tripla, emergidos com 15 dias

Table 3 -Alimentary preference of larvae in substrata with $2 g$ of maize flour, wheat and integral soybean in the Petri plate with triple thick partition wall emerged with 15 days

\section{Tipos de Substratos}

Milho

Trigo

Soja

\section{№ médio de descendentes por indivíduos}

$\begin{aligned} 60,60 & \pm 4,14 \text { (a) } \\ 33,70 & \pm 4,56 \text { (b) } \\ 8,87 & \pm 8,27 \text { (c) }\end{aligned}$

${ }^{*}$ Letras diferentes indicam diferenças estatísticas (Tukey $P \geq 0,05$ ).

entre desempenho e preferência larval.

As larvas emergiram primeiro no substrato farinha de trigo e farinha de milho, chegando a medir 5-7 $\mathrm{mm}$ de comprimento e posteriormente no substrato na farinha de soja integral, chegando a medir 2-4 mm de comprimento.

O número médio de descendentes adultos é um dado que correlaciona dois parâmetros muito significativos, utilizados para medir o desempenho, ou seja, a fecundidade das fêmeas e a porcentagem de emergência dos ovos colocados. Em outras palavras, o resultado final do desempenho da fêmea revela em grande parte o seu sucesso reprodutivo, ou seja, a quantidade de descendentes (FOX, 1993).

Esta etapa do experimento é um dado importante, pois considera apenas os indivíduos que chegaram à idade reprodutiva, completando o ciclo entre 21-28 dias.

O número médio de adultos emergentes, ou seja, de insetos potencialmente reprodutores, é um dado importante para medir o sucesso de uma população. Houve menor 
emergência de adultos na farinha de soja integral, sendo que o ciclo de vida na farinha de trigo e na farinha de milho completou em 21 dias e na farinha de soja integral completou entre 23-28

TABELA 4 - Preferência Alimentar dos adultos nos substratos com $2 \mathrm{~g}$ de farinha de milho, trigo e soja integral na placa de Petri sem divisória, emergidos com 21 dias

Table 4 -Alimentary preference of the adults in substrata with $2 \mathrm{~g}$ of maize flour, wheat and integral soybean in the Petri plate without thick partition wall emerged with 21 days

\section{Tipos de Substratos}

Milho

Trigo

Soja

*Letras diferentes indicam diferenças estatísticas (Tukey $P \geq 0,05$ ).

\section{№ médio de descendentes por indivíduos}
$5,3 \pm 0,57$ (a)
$3,0 \pm 0,55(\mathrm{~b})$
$0,9 \pm 0,23(\mathrm{c})$

dias. $\quad$ Entretanto, esse dado pode ser resultado da aceitabilidade do Tribolium castaneum na farinha de soja integral.

Vários fatores ecológicos, genéticos e fisiológicos contribuem para a evolução e manutenção da amplitude da dieta de um inseto. A capacidade de dispersão da larva por si só indica que o comportamento da seleção de hospedeiros é um importante determinante na limitação do número de hospedeiros utilizados (TEIXEIRA; ZUCOLOTO, 2003).

As fêmeas de Tribolium castaneum que se desenvolveram em um determinado hospedeiro são perfeitamente capazes de ovipositar em outro hospedeiro, desde que esse contenha os componentes nutricionais que são necessários ao desenvolvimento da prole (PIMBERT, 1985; OFUYA; CREDLAND, 1996). No entanto, o reconhecimento de um possível hospedeiro está associado a substâncias específicas, que muitas vezes são chamadas de estímulo-sinal (BERNAYS, 1998).

Pode-se observar no experimento que a distribuição de larvas nos substratos ocorreu de maneira particular. As larvas emergiram primeiro no substrato farinha de trigo e farinha de milho, medindo até 5-7 mm de comprimento, e posteriormente no substrato na farinha de soja integral, medindo até $2-4 \mathrm{~mm}$ de comprimento.

O número de descendentes de uma fêmea pode ser considerado conseqüência final do desempenho dessa e bom parâmetro para se medir o desempenho numa determinada situação.

Ao analisar a preferência alimentar, percebe-se que não existe relação entre preferência e desempenho nesses hospedeiros. Uma possível explicação é que essa relação entre Tribolium castaneum e o hospedeiro farinha de soja integral é nova.

Os resultados obtidos neste trabalho permitem reforçar a importância da preferência alimentar do Tribolium castaneum na farinha de milho, considerando-o como seu principal hospedeiro. Também permitiu observar que esta especialização não limita o desempenho do Tribolium castaneum, pois a preferência alimentar pode sofrer alterações de acordo com o regime de seleção, o que permite que o inseto se adapte a diferentes situações de disponibilidade de alimento.

A temperatura e a umidade constituem elementos determinantes na ocorrência de insetos e têm grande influência no seu desenvolvimento, favorecendo um elevado potencial reprodutivo. Diante destes dados, conclui-se que a estabilidade da umidade, temperatura e local adequado livre de sujidade são fundamentais para o controle preventivo da ocorrência dos besouros.

\section{CONCLUSÕES}

Com base nos resultados e nas condições em que foi realizado este experimento, pode-se concluir que: 
- Houve menor aceitabilidade na farinha de soja integral e uma correlação positiva entre desempenho e preferência larval, ocorrendo grande variação entre os substratos.

- As larvas emergiram inicialmente no substrato farinha de trigo e farinha de milho, chegando posteriormente ao substrato farinha de soja integral.

- A distribuição de larvas nos substratos ocorreu de maneira singular.

- Não foi observada uma relação entre preferência alimentar e desempenho nos hospedeiros.

Os resultados obtidos neste trabalho permitem reforçar a importância da preferência alimentar do Tribolium castaneum na farinha de milho, considerando-o como seu principal hospedeiro. Também permitiu observar que esta especialização não limita o desempenho do Tribolium castaneum, pois a preferência alimentar pode sofrer alterações de acordo com o regime de seleção, o que permite que o inseto se adapte a diferentes situações de disponibilidade de alimento.

Diante destes dados, conclui-se que a estabilidade da umidade, temperatura e local adequado livre de sujidade são fundamentais para o controle preventivo da ocorrência deste tipo de pragas em alimentos processados.

\section{REFERÊNCIAS}

ANVISA. Agência de Vigilância Sanitária. Legislação. 2001. Disponível em: <http://www.anvisa.gov.br>. Acesso em: 23 nov. 2003.

BERNAYS, E. A. Evolution of feeding behavior in insects herbivores. BioScience, v. 48, n. 1, p. 35-44. Jan. 1998.

EMBRAPA SOJA. Tecnologias de produção de soja: Região Central do Brasil. 2003. Disponível em: <http:/ /www.cnpso.embrapa.br>. Acesso em: 14 jul. 2003.

FOX, C. W. Maternal and genetic influences on eggs size and larval performance in a seed beetle (Callosobruchus maculates): jultigenerational transmition of a maternal effect. Heredity, 1993.

LORINI, I.; MIIKE L. H; SCUSSEL V. M. Armazenagem de grãos. Campinas: IBG, 2002.

OFUYA, T. I.; CREDLAND, P. F. The ability of Bruchidius atrolineatus (PIC)(Coleoptera: Bruchidae) to infest and damage seeds of different tropical legumes. Journal of Stored Products Research, v. 32, n. 4, p. 323328, 1996.

PIMBERT, M. P. Reproduction and oviposition preferences of Zabrotes subfasciatus stocks reared from two host plant species. Entomologia Experimentalis et Applicata, v. 38, n. 3, p. 273-276, 1985.

TEIXEIRA, I. R. V; ZUCOLOTO, F. S. Seed suitability and oviposition behaviour of wild and selected populations of zabrotes subfasciatus (Boheman) (Coleoptera, Bruchidade) on different hosts. Journal of Stored Products Research, v. 39, p. 131-140, 2003. 